\title{
miR-125a-5p Regulates Osteogenic Differentiation of Human Adipose-Derived Mesenchymal Stem Cells under Oxidative Stress
}

\author{
Yongheng Ye $\mathbb{D},{ }^{1}$ Quan Liu, ${ }^{2}$ Changzhao Li $\mathbb{D}^{1},{ }^{1}$ and Peiheng He $\mathbb{D}^{1}$ \\ ${ }^{1}$ Department of Joint Surgery, The First Affiliated Hospital of Sun Yat-sen University, Guangzhou 510080, China \\ ${ }^{2}$ Department of Orthopaedic Surgery, The First People's Hospital of Nankang, Ganzhou 341400, China \\ Correspondence should be addressed to Peiheng He; hepeiheng@mail.sysu.edu.cn
}

Received 12 January 2021; Revised 9 March 2021; Accepted 23 May 2021; Published 7 June 2021

Academic Editor: Xinhua Li

Copyright ( 2021 Yongheng Ye et al. This is an open access article distributed under the Creative Commons Attribution License, which permits unrestricted use, distribution, and reproduction in any medium, provided the original work is properly cited.

\begin{abstract}
Adipose-derived mesenchymal stem cells (ADSCs) are a well-recognized multilineage stem cell with vital clinical feasibility for tissue regeneration. Extensive evidence indicates that oxidative stress and microRNAs (miRNAs/miRs) play an important role in the osteoinduction of adipose-derived mesenchymal stem cells. In this study, we investigated the mechanism of miR-125a-5p in regulating the osteogenesis of human adipose-derived mesenchymal stem cells (hADSCs) under oxidative stress. The expression of miR-125a-5p lessened gradually during the osteogenic differentiation of hADSCs. Relative to the negative group, the expression levels of runt-related transcription factor 2 (RUNX2), alkaline phosphatase (ALP), osteocalcin (OCN), and osterix in the miR-125a-5p group were marked lower than those in the miR-125a-5p inhibitor group. The levels of p16, p21, p53, miR-125a-5p, and ROS during osteoinduction of hADSCs were assessed in vitro under oxidative stress and were observed to be upregulated. Further experiments showed that oxidative stress and miR-125a-5p together suppressed the expression of VEGF during osteogenic differentiation of hADSCs and that the inhibition of miR-125a-5p reversed the effect of oxidative stress. In short, our study indicated that miR-125a-5p is induced under oxidative stress and inhibits the expression of VEGF, leading to the reduction of osteogenic differentiation of hADSCs. Our outcomes showed that miR-125a-5p could be a potential clinical target for bone repairing.
\end{abstract}

\section{Introduction}

Bone loss caused by trauma, tumor, and age-related osteoporosis is a common problem in orthopedics. The balance of bone metabolism is a dynamic course influenced by osteoblasts, osteoclasts, and osteocytes. Oxidative stress, causing many reactive oxygen species (ROS) to form, plays an important role in the development of skeletal diseases [1-3]. The activity of oxidants has also been proven to enhance osteoclast differentiation [4]. With the need for more effective treatment, it is of great significance to find osteogenic methods for bone repair and regeneration.

Stem cells can differentiate into the bone, cartilage, and fat under the stimulation of certain factors, providing promising therapeutic applications with regard to the restoration of tissue defects [5-7]. Many researches have focused on efforts to utilize bone mesenchymal stem cells (BMSCs) for bone tissue engineering [8]. In addition, previous studies have proven that VEGF regulates adipocyte and osteoblast differentiation in mesenchymal stem cells [9]. Adiposederived mesenchymal stem cells (ADSCs) as seed cells are more readily available to that of BMSCs, but their osteogenic differentiation ability is limited [10]. Aging is the main factor leading to decreased activity and repair ability of mesenchymal stem cells [11]. Oxidative stress is one of the main causes of aging. There is evidence to show that oxidative stress suppresses osteogenic differentiation and weakens the stemness of MSCs, which causes bone formation defects [12-14]. In addition, oxidative stress can induce apoptosis, senescence, and death of MSCs [15]. Therefore, clarifying the regulating mechanisms of osteogenic differentiation of ADSCs is helpful for identifying their possible therapeutic applications in bone repair.

MicroRNA (miRNA) is a single stranded RNA molecule consisting of about 18-22 nucleotides, which play a key role in cell apoptosis, proliferation, tumorigenesis, and other physiological and pathological processes [16]. Several 
investigations have shown that miRNAs are mentioned in the osteoblast differentiation of stem cells. For example, miR-199a-5p controls the osteogenic induction of bone marrow mesenchymal stem cells by targeting TET2 [17]. In addition, miR-450b promotes osteoblast induction in vitro and strength bone generation in vivo by targeting the BMP3 signaling pathway [18]. Prior studies indicate that the downregulation of miR-125a-5p was detected during osteogenic differentiation of hADSCs by microarray profiles of small RNA molecules [19]. However, by far, the regulating mechanism of miR-125a-5p in the osteogenic differentiation of hADSCs has not been reported.

Therefore, the purpose of this research was to investigate the specific mechanisms of miR-125a-5p controlling osteogenesis in hADSCs under oxidative stress. It is expected that our study will provide a beneficial groundwork for the application of miR-125a-5p in bone restoration.

\section{Materials and Methods}

2.1. Isolation and Culture of Cells. The human adiposederived mesenchymal stem cells were purchased from Cyagen, USA (No. HUXMD-01001). The hADSCs were cultured in Dulbecco's Modified Eagle Medium: Nutrient Mixture F-12 (DMEM/F12, Gibco) medium, which includes $10 \%$ fetal bovine serum (FBS, Sigma), $100 \mathrm{U} / \mathrm{mL}$ penicillin, and $100 \mu \mathrm{g} / \mathrm{mL}$ streptomycin, cultured incubated in a $5 \%$ $\mathrm{CO}_{2}$ atmosphere at $37^{\circ} \mathrm{C}$. The complete medium was changed in $48-72 \mathrm{~h}$, and the cells were passaged when $70 \sim 90 \%$ confluence was reached. hADSCs were expanded until passage 3 .

2.2. hADSC Osteogenic Differentiation. The cells (passage 3) were transplanted to 6-well cell culture plates. Cells were grown to $50-70 \%$ confluence in complete medium after 24-48 h [20]. The medium was completely replaced with an osteogenic differentiation medium, which consisted of human adipose-derived stem cell osteogenic differentiation basal medium, human adipose-derived stem cell osteogenic differentiation fetal bovine serum, penicillin-streptomycin, glutamine, ascorbate, $\beta$-glycerophosphate, and dexamethasone (Cyagen, USA). The cells were then cultured up to 21 days. The osteogenic medium was changed every $72 \mathrm{~h}$.

2.3. Reactive Oxygen Species (ROS) Measurement. Cells were transplanted into $2 \times 10^{4}$ cells $/ \mathrm{cm}^{2}$ in 6 -well plates. After $24 \mathrm{~h}, 50,100$, and $200 \mu \mathrm{M} \mathrm{H}_{2} \mathrm{O}_{2}$ were added into the fresh medium to stimulate oxidative stress response, and cells were incubated for another $24 \mathrm{~h}$. Then, the cells were incubated in a new solution without the FBS medium and including 10 $\mu \mathrm{M}$ of the Reactive Oxygen Species Assay Kit (ROS Assay Kit, Beyotime, China) for $0.5 \mathrm{~h}$. Finally, $1000 \times g$ centrifugation of cells was conducted for $5 \mathrm{~min}$ and they were then washed with phosphate-buffered saline (PBS) for 2-3 times. The levels of dichlorofluorescein (DCF) were tested by flow cytometry (CytoFLEX, Beckman Coulter, USA) as prescribed by the manufacturer's instructions.

2.4. Quantitative Real-Time Polymerase Chain Reaction ( $q R T-P C R)$. hADSCs were lysed and gathered using the
TABle 1: Primer sequences for qRT-PCR.

\begin{tabular}{lc}
\hline Gene or miRNA & Sequence $\left(5^{\prime}\right.$ to $\left.3^{\prime}\right)$ \\
\hline miR-125a-5p & F: CGATTCCCTGAGACCCTTTAA \\
ALP & F: CGAGATACAAGCACTCCCACTTC \\
& R: CTGTTCAGCTCGTACTGCATGTC \\
Funx2 & R: GTGGTAGAGTGGATGGAC \\
& F: GGTGCAGCCTTTGTGTCCAAGC \\
OCN & R: GTCAGCCAACTCGTCACAGTCC \\
& F: TGCTTGAGGAGGAAGTTC \\
Osterix & R: CTTTGCCCAGAGTTGTTG \\
p16 & F: GATCCAGGTGGGTAGAAGGTC \\
& R: CCCCTGCAAACTTCGTCCT \\
p21 & F: TGTCCGTCAGAACCCATGC \\
& R: AAAGTCGAAGTTCCATCGCTC \\
p53 & F: CAGCACATGACGGAGGTTGT \\
& R: TCATCCAAATACTCCACACGC \\
GAPDH & F: CCATCTTCCAGGAGCGAGATC \\
& R: GCCTTCTCCATGGTGGTGAA \\
U6 & F: CTCGCTTCGGCAGCACA \\
& R:AACGCTTCACGAATTTGCGT \\
\hline
\end{tabular}

TRIzol Reagent (Invitrogen, USA) according to the manufacturer's instructions. cDNA was synthesized using reverse transcription with a PrimeScript RT reagent kit (Takara, Dalian, China) and amplified using $2 \times$ SYBR Green $\mathrm{qPCR}$ Master Mix (TOYOBO, Japan) as prescribed by the manufacturer's instructions. Primers of human osteoblastic marker genes, miR-125a-5p, and U6 were compounded by RIBOBIO (Guangzhou, China). The primers for qRT-PCR are shown in Table 1. U6 and GAPDH were the reference genes of miRNA and mRNA, respectively. Relative expression levels were quantified by the $2^{-\Delta \Delta \mathrm{CT}}$ method. Each experiment was repeated 3 times, and each assay was performed in triplicate.

2.5. Cell Counting Kit-8 (CCK-8) Assay. Cell viability was measured by the CCK- 8 colorimetric assay. hADSCs $\left(3 \times 10^{3} /\right.$ well $)$ were cultured to confluence on 96 -well culture plates and incubated with increasing concentrations of $\mathrm{H}_{2} \mathrm{O}_{2}$ $(50,100$, and $200 \mu \mathrm{M})$ and increasing time $(4,6,8$, and $24 \mathrm{~h})$. After treatment, the cells were incubated with $10 \mu \mathrm{L}$ CCK-8 for $0.5-4 \mathrm{~h}$, and the absorbance was measured at $450 \mathrm{~nm}$ using a microplate reader (Sunrise, TECAN, Austria).

2.6. Western Blotting. The target protein productions were gathered in RIPA lysis buffer (Solarbio, China), including the protease inhibitor, and put on ice for $15 \mathrm{~min}$. After processing through electrophoresis and membrane transferring, the membranes were incubated by the anti-VEGF $(1: 1000$ dilution, ab32152, Abcam, Cambridge, UK) antibody. After washing, the membranes were incubated with the corresponding secondary antibody (Proteintech, Wuhan, China) as prescribed by the manufacturer's instructions. Protein bands were quantified using the ImageJ software (National Institutes of Health, Bethesda, MD). 


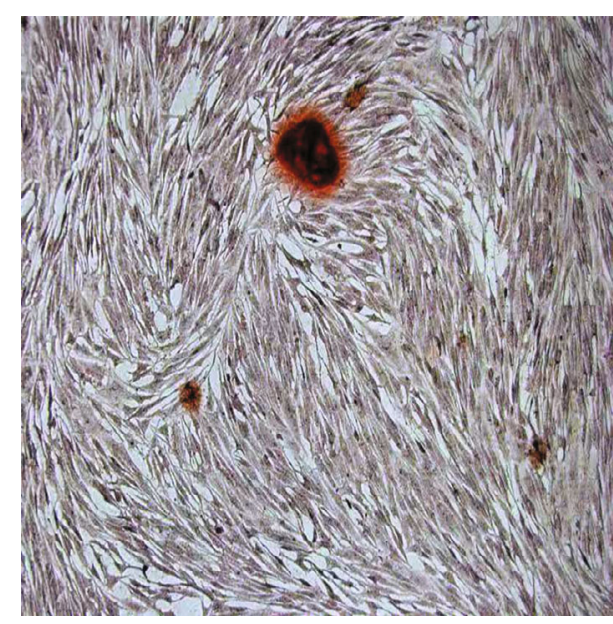

(a)

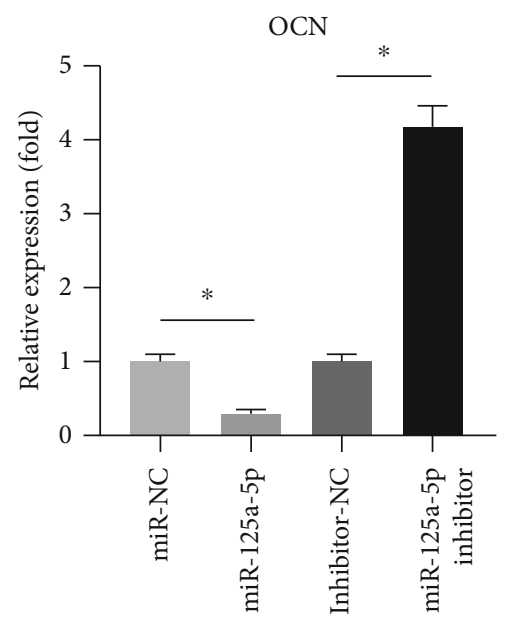

miRNA-125a-5p

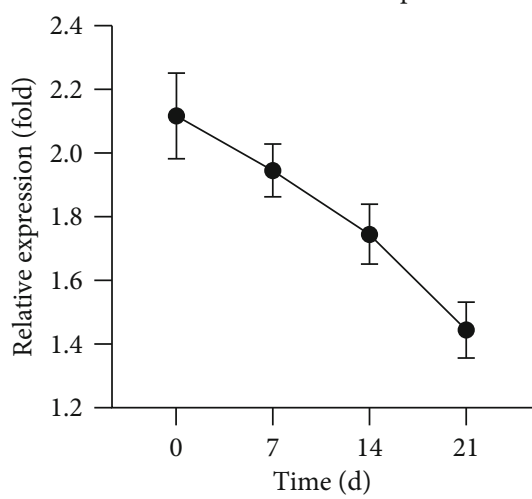

(c)

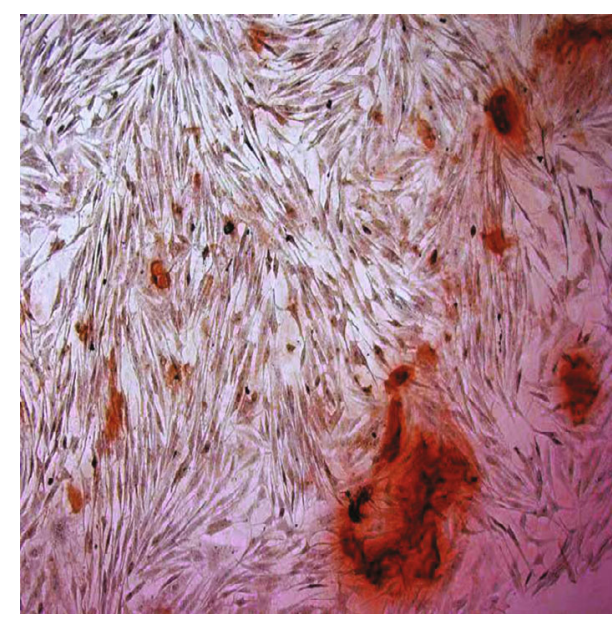

(b)

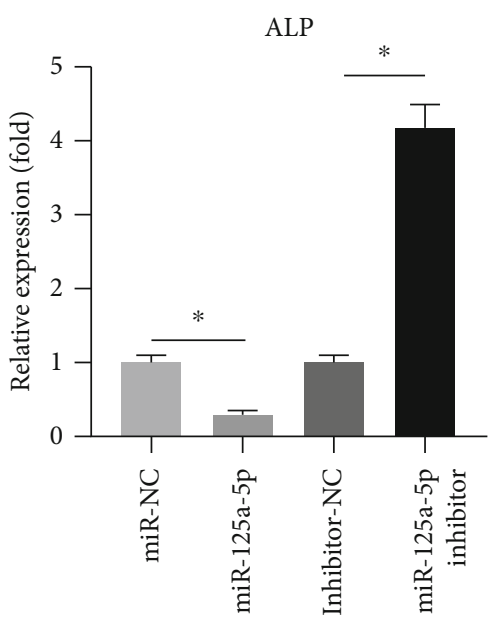

Osterix
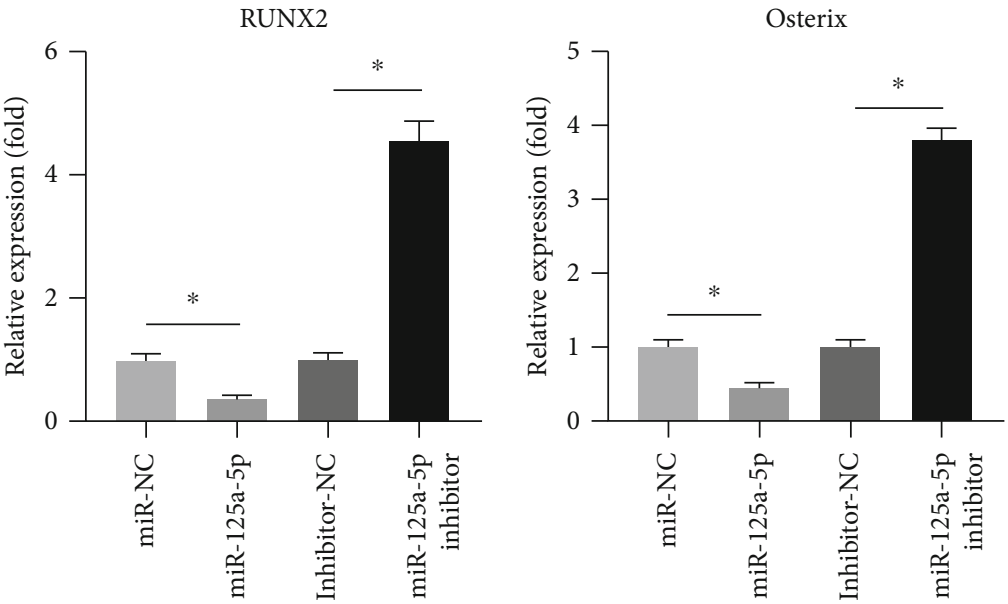

(d)

FIGURE 1: miR-125a-5p inhibits the osteogenic differentiation of hADSCs. (a, b) Osteogenic differentiation was induced in hADSCs on days 14 and 21 and was then processed with Alizarin red staining. (c) After the induction of osteogenic differentiation, the relative expression of miRNA-125a-5p was measured on days $0,7,14$, and 21. (d) mRNA relative expression of the osteoblastic marker genes OCN, ALP, RUNX2, and osterix were measured after being transfected with miRNA-125a-5p and miRNA-125a-5p inhibitor. $N=3 ; *$ means $p<0.05 ; * *$ means $p<0.01$. 


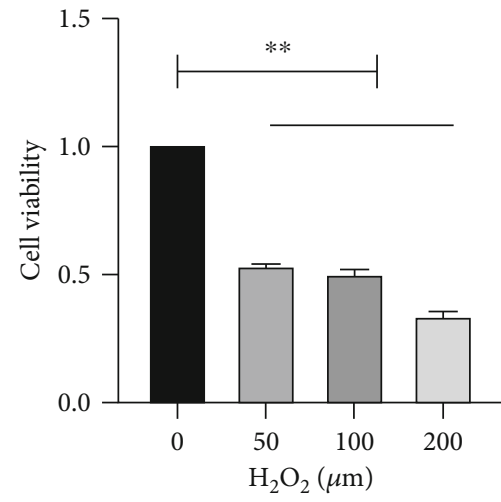

(a)

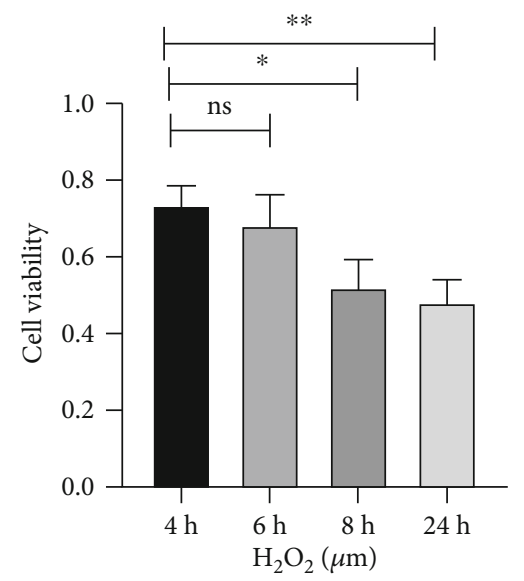

(b)

Figure 2: Effects of $\mathrm{H}_{2} \mathrm{O}_{2}$ on the viability of hADSCs. $(\mathrm{a}, \mathrm{b})$ Cell viability was measured by the CCK- 8 colorimetric assay in different concentrations of $\mathrm{H}_{2} \mathrm{O}_{2}$ and induction times. $N=3$; ${ }^{\mathrm{ns}} p>0.05 ;{ }^{*} p<0.05 ;{ }^{* *} p<0.01$.

2.7. Cell Transfection. After $24 \mathrm{~h}$ of seeding, the miR-125a-5p mimics and miR-125a-5p inhibitor were transfected into the cells by the Lipofectamine ${ }^{\mathrm{TM}} 2000$ transfection reagent as prescribed by the manufacturer's instructions (Gibco Life Technologies, USA); miR-NC and inhibitor-NC were used as the control and negative control, respectively. The medium was replaced after $24 \mathrm{~h}$, and the cells were gathered for subsequent experiments.

2.8. Alizarin Red Staining (ARS). hADSCs or hADSCs transfected with miR-125a-5p mimic (50 nM), miR-125a-5p inhibitor $(50 \mathrm{nM})$, and corresponding negative control were seeded into 24-well plates, respectively. After reaching 75$90 \%$ density, the hADSCs were induced using the osteogenic differentiation medium for a further 3 weeks. After 14 and 21 days, the hADSCs were fixed by the $4 \%$ Paraformaldehyde and stained with ARS for $15 \mathrm{~min}$ at $23 \pm 2^{\circ} \mathrm{C}$, followed by analysis under an inverted microscope (Leica DMI4000B, Leica, Germany).

2.9. Statistical Analysis. SPSS 19.0 software (IBM Corporation, Armonk, NY, USA) was used for the statistical analysis. Data are presented as the mean \pm standard deviation (mean $\pm \mathrm{SD}$ ). Comparisons between groups were used oneway analysis of variance followed by Tukey's post hoc test. A $p$ value $<0.05$ was treated as significant.

\section{Results}

3.1. miR-125a-5p Inhibits Osteogenic Differentiation of hADSCs. The presence of calcium deposition in hADSCs indicates that osteogenic differentiation was induced in hADSCs (Figures 1(a) and 1(b)). We first detected the expression level of miR-125a-5p in hADSCs by qRT-PCR in the osteogenic differentiation of hADSCs. The change of miR-125a-5p was reduced on day 7 compared with day 0 and continuously decreased to day 21 (Figure 1(c)). This suggested that miR-125a-5p might negatively regulate the osteogenic differentiation of hADSCs. hADSCs were transfected with the miR-125a-5p mimic and the miR-125a-5p inhibitor. The expression level of osteogenic marker genes was evaluated by qRT-PCR for osteogenic differentiation in 21 days. The results show that the mRNA level of the osteogenic marker genes was reduced after being transfected with the miR-125a-5p mimic, whereas the level expression of the osteogenic marker genes was increased after being transfected with the miR-125a-5p inhibitor (Figure 1(d)). It can be seen from these results that miR-125a-5p negatively regulated the osteogenic differentiation of hADSCs.

3.2. Effects of $\mathrm{H}_{2} \mathrm{O}_{2}$ on Cell Viability. The cytotoxic effect of $\mathrm{H}_{2} \mathrm{O}_{2}$ on hADSC viability was evaluated at 50,100, and $200 \mu \mathrm{M}$ for $4,6,8$, and $24 \mathrm{~h}$ using a CCK-8 assay. As shown in Figure 2, the cell viability was deceased by $50 \%$ with the $100 \mu \mathrm{M} \mathrm{H}_{2} \mathrm{O}_{2}$ and for $24 \mathrm{~h}$.

3.3. Oxidative Stress Induces Senescence of hADSCs and miR125a-5p Expression. thADSCs were stimulated by different doses of $\mathrm{H}_{2} \mathrm{O}_{2}$ for $24 \mathrm{~h}$ to simulate the environment of oxidative stress response. As can be seen in Figures 3(a)-3(c), the three-cell senescence-related markers p16, p21, and p53 were significantly increased along with the dose of $\mathrm{H}_{2} \mathrm{O}_{2}$ and induction time. Furthermore, we can find that the level of ROS was increased along with the dose of $\mathrm{H}_{2} \mathrm{O}_{2}$, which was marked at $100 \mu \mathrm{M}$ and $200 \mu \mathrm{M}$, and $100 \mu \mathrm{M} \mathrm{H}_{2} \mathrm{O}_{2}$ was chosen for subsequent experiments (Figure 3(d)). The expression level of miR-125a-5p was increased following an increase in the concentration of $\mathrm{H}_{2} \mathrm{O}_{2}$ (Figure 3(e)). These data suggested that hADSCs exhibit an obvious increase in senescence with oxidative stress, which was the trigger to increase the expression level of miRNA-125a-5p.

3.4. Oxidative Stress Increases miR-125a-5p Expression during Osteogenic Differentiation of hADSCs. It was observed that the osteogenic marker genes continually increased when osteogenic differentiation was induced in hADSCs from day 0 to day 21. However, when osteogenic differentiation was induced in hADSCs in the presence of $100 \mu \mathrm{M} \mathrm{H}_{2} \mathrm{O}_{2}$, the 


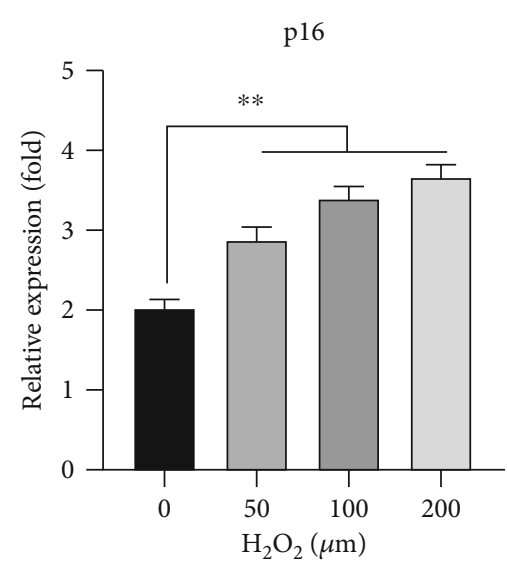

(a)

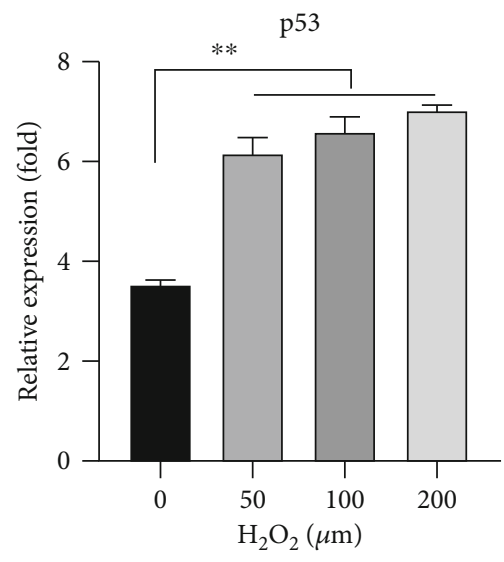

(c)

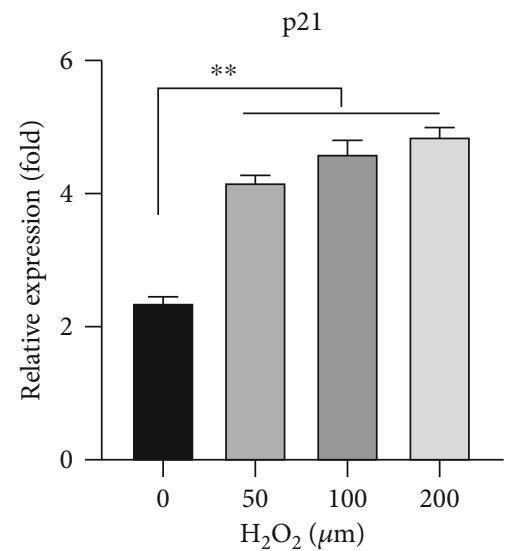

(b)

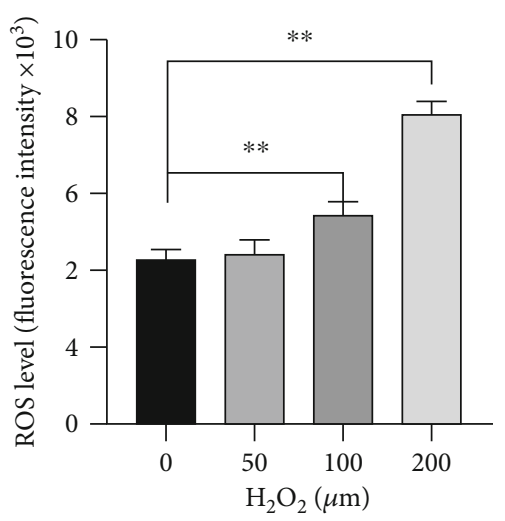

(d)

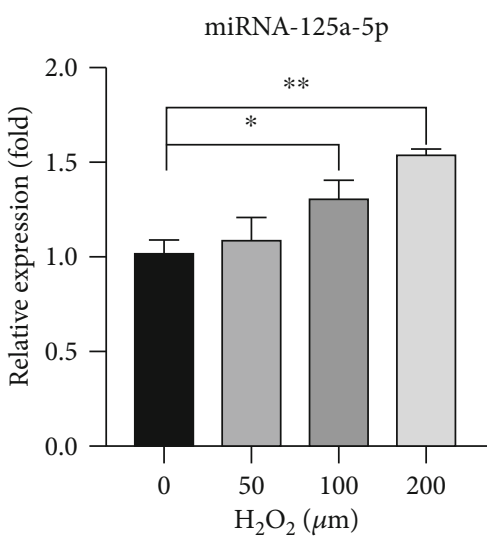

(e)

FIGURE 3: Oxidative stress induces senescence of hADSCs and miR-125a-5p expression. (a-c) mRNA expression levels of p16, p21, and p53 were evaluated by qRT-PCR. (d) The ROS level of the cells was measured by exposure to different concentrations of $\mathrm{H}_{2} \mathrm{O}_{2}$. (e) The expressions of miRNA-125a-5p were measured by exposure to different concentrations of $\mathrm{H}_{2} \mathrm{O}_{2} . N=3 ;{ }^{*} p<0.05 ;{ }^{* *} p<0.01$.

expression level of osteogenic marker genes decreased (Figure 4(a)). The level of miRNA-125a-5p was reduced in osteogenic differentiation, but the level in the medium with $\mathrm{H}_{2} \mathrm{O}_{2}$ was higher than that in the medium without $\mathrm{H}_{2} \mathrm{O}_{2}$ (Figure 4(b)). These results show that oxidative stress increased miR-125a-5p and concomitantly reduced osteogenic differentiation during the osteogenic differentiation of hADSCs.
3.5. miR-125a-5p Mediates the VEGF-Regulated Osteogenic Differentiation of hADSCs under Oxidative Stress. By using the web-based bioinformatics tool, miRBase (http://mirtarbase .cuhk.edu.cn), a predictor gene is considered a target gene of miRNA when it met software's requirement. It was found that miR-125a-5p's regulated target gene is VEGF. Thus, we first analyzed VEGF levels in transfected cells and found that the VEGF proteins were significantly declined with transfected 

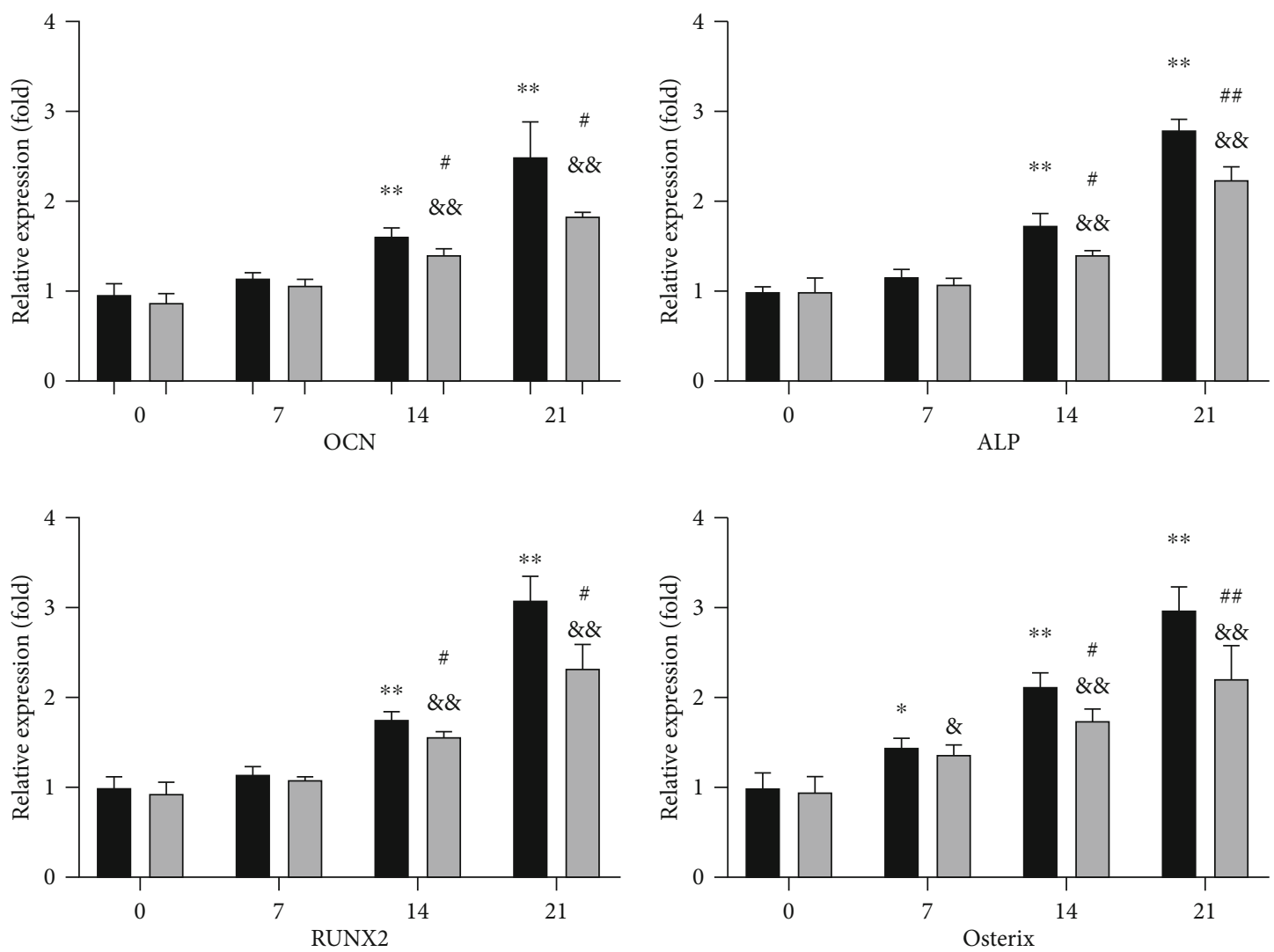

ODM

$\mathrm{ODM}+\mathrm{H}_{2} \mathrm{O}_{2}$

(a)

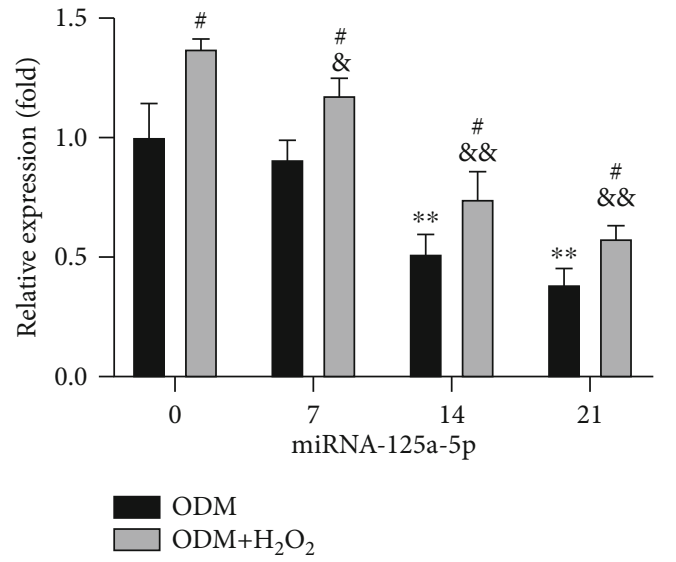

(b)

FIGURE 4: Oxidative stress increases miR-125a-5p expression during osteogenic differentiation of hADSCs. (a, b) The expression levels of the osteoblastic marker genes and the miRNA-125a-5p were measured with or without $\mathrm{H}_{2} \mathrm{O}_{2}$ in the condition of osteogenic differentiation medium (ODM). $N=3 ;{ }^{*} p<0.05,{ }^{* *} p<0.01$ vs. day 0 (ODM group); ${ }^{*} p<0.05,{ }^{\#} p<0.01$ vs. day $0\left(\mathrm{ODM}+\mathrm{H}_{2} \mathrm{O}_{2}\right.$ group); ${ }^{*} p<0.05$, $\& \& p<0.01\left(\mathrm{ODM}+\mathrm{H}_{2} \mathrm{O}_{2}\right.$ group vs. ODM group)

miR-125a-5p mimic and rose with transfected miR-125a-5p inhibitor (Figure 5(a)). Then, hADSCs were cultured under osteogenic conditions with $100 \mu \mathrm{M} \mathrm{H}_{2} \mathrm{O}_{2}$, and the mRNA level of VEGF was evaluated by qRT-PCR at different time points during osteogenic differentiation. Although the mRNA level of VEGF showed marked increase during osteogenic differentiation up to 21 days, the VEGF levels were decreased when $\mathrm{H}_{2} \mathrm{O}_{2}$ stimulated the oxidative stress response (Figure 5(b)). Furthermore, under the conditions of oxidative stress, the overexpression of miRNA-125a-5p could suppress the expression of VEGF while the overexpression of miRNA-125a-5p inhibitor could promote the expression of VEGF (Figure 5(c)). These results provide strong evidence that miR-125a-5p could mediate the expression level of VEGF. 


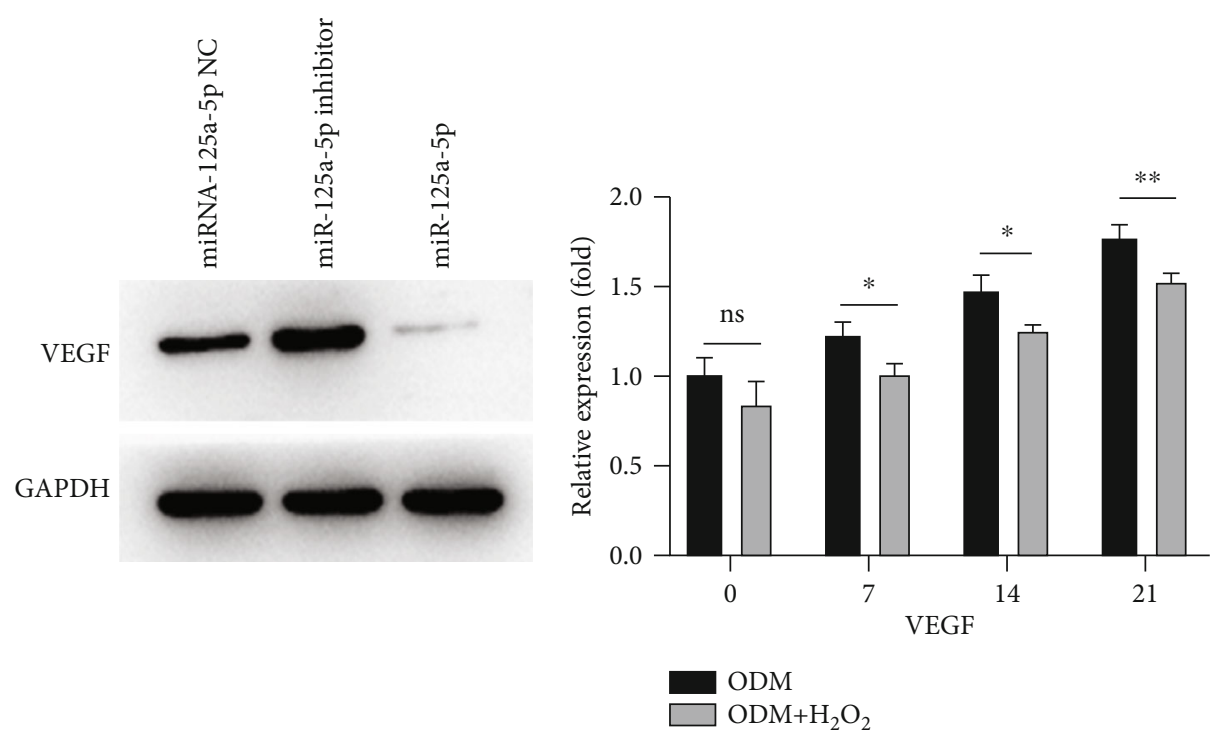

(a)

(b)

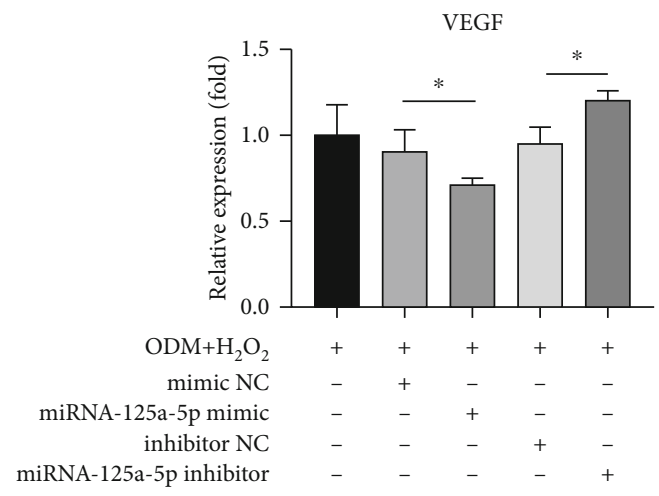

(c)

FIGURE 5: miR-125a-5p mediates the VEGF-regulated osteogenic differentiation of hADSCs under oxidative stress. (a) The expression level of VEGF protein in hADSCs infected by miRNA-125a-5p or infected with miRNA-125a-5p inhibitor was measured by western blotting. (b) The expression level of VEGF was evaluated at different time points during osteoinduction with or without $\mathrm{H}_{2} \mathrm{O}_{2}$. (c) The expression level of VEGF was measured by qRT-PCR on day 21 , and after that, the cells were induced by the osteogenic differentiation medium (ODM) and, with the $\mathrm{H}_{2} \mathrm{O}_{2}$ stimulation, infected by miRNA-125a-5p or infected with miRNA-125a-5p inhibitor. $N=3 ;{ }^{\mathrm{ns}} p>0.05 ;{ }^{*} p<0.05$.

\section{Discussion}

ADSCs are found largely and can be obtained readily, and they have the ability to differentiate into numerous cell lineages, including hepatocytes, endothelial cells, smooth muscle cells, cardiomyocytes, neurons, adipocytes, osteocytes, and chondrocytes. Therefore, ADSCs are known as a tool for repairing, replacing, and regenerating damaged tissue, but the limited osteogenic differentiation ability greatly impedes the clinical application of ADMSCs in bone repair [21].

Specific regulatory factors and multiple signaling pathways are involved in the progression of osteogenesis [22, 23]. miRNAs have been shown to have a vital role in osteogenic differentiation and may become vital for regulating bone repair $[24,25]$. The role of $\mathrm{miR}-125 \mathrm{a}-5 \mathrm{p}$ has been researched in a variety of cell lineages. As a functional microRNA, miR-125a-5p regulates the progression of bladder cancer by effecting on FUT4 [26]. Recently, a study has revealed that increased miR-125a-5p decreases the expression of
TNFRSF1B and increases osteoclast differentiation [27]. The outcomes of previous studies suggest that miR-125a-5p affects the osteogenic differentiation of ADSCs [19]. This current study further explored the specific mechanism of miR$125 \mathrm{a}-5 \mathrm{p}$ in the osteogenic differentiation of hADSCs. We show an obvious decrease in miR-125a-5p expressions during the osteogenic differentiation of hADSCs. Meanwhile, the overexpression of miR-125a-5p suppressed osteogenic induction of hADSCs, and the inhibition of miR-125a-5p by transfecting inhibitor oligonucleotide increased osteogenesis. Taken together, these outcomes show that miR-125a-5p is a negative regulator of the osteogenic differentiation of hADSCs.

Senescence of MSCs can be caused by different stimuli, including oxidative stress, radiation, chemicals (inducing acute senescence), or replication failure (inducing chronic senescence) [28]. Oxidative stress is induced by an imbalance between an excessive reactive oxygen species (ROS) and lacking antioxidant defense mechanisms [29]. The properties and their linked differentiation of ADSCs are regulated by 


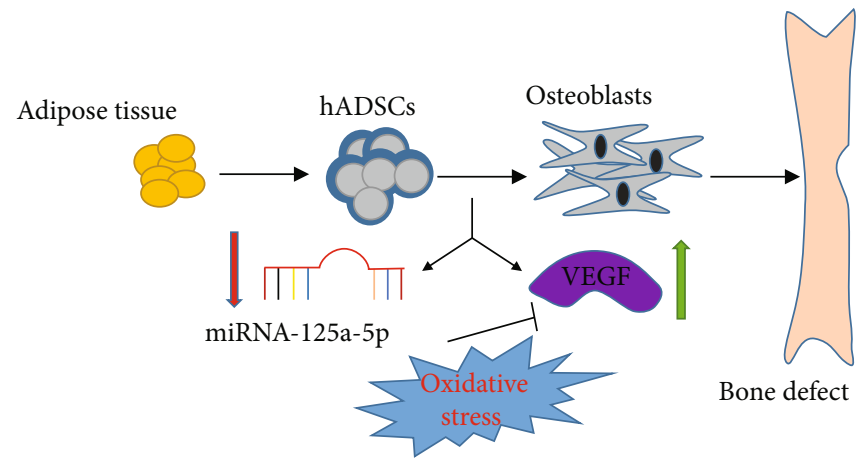

FIGURE 6: Schematic illustration of miRNA-125a-5p as it regulates osteogenic differentiation of hADSCs under oxidative stress by inhibiting the expression of VEGF.

reactive oxygen species in some degree [30]. In this study, hADSCs were treated with $100 \mu \mathrm{M} \mathrm{H}_{2} \mathrm{O}_{2}$ to mimic the environment of oxidative stress and induce the generation of ROS. When hADSCs were grown in osteogenic differentiation medium, the levels of osteogenic genes exhibited a timedependent increase. However, the osteogenic genes tended to be reduced by oxidative stress stimulation. Meanwhile, under oxidative stress, ROS, the senescence of hADSCs, and miR$125 a-5 p$ were increased over time, suggesting that oxidative stress suppresses the osteogenic differentiation of hADSCs, induces the senescence of hADSCs, and is important for miR-125a-5p production.

Researchers have shown that VEGF has dual activities of angiogenesis and osteogenesis, which can significantly promote the growth of osteoblasts and accelerate bone formation [31]. Previous studies have also found that lamin A/C regulates the transcription factors Runx 2 and PPAR $\gamma 2$ by VEGF to control the osteogenic or adipogenic differentiation of mesenchymal stem cells. Moreover, the reduction of osteogenic differentiation of MSCs caused by VEGF gene deletion could only be restored by retrovirus-mediated VEGF expression, rather than by the exogenous recombination of VEGF [9]. In this study, it was proven using western blotting that miR-125a-5p controls the expression of VEGF in hADSCs. Meanwhile, we also showed that oxidative stress has a key role in regulating VEGF during the osteogenic differentiation of hADSCs. Oxidative stress and miR-125a-5p together suppressed the production of VEGF in the course of osteogenic differentiation of hADSCs, and the effect of oxidative stress could be reversed by the inhibition of miR-125a-5p.

In conclusion, our study pointed out that miR-125a-5p is induced under oxidative stress and inhibits the expression of VEGF, leading to the reduction of osteogenic differentiation of hADSCs (Figure 6). This demonstrates that miR-125a-5p could be a potential clinical target for bone repairing.

\section{Data Availability}

All experiment methods and the result generated or used during the study appear in the submitted article.

\section{Conflicts of Interest}

The authors have declared that no competing interest exists.

\section{Authors' Contributions}

Yongheng Ye and Quan Liu contributed equally to this work.

\section{Acknowledgments}

This work was supported by the Guangdong Natural Science Foundation (No. 2016A030313259).

\section{References}

[1] A. J. Serra, J. R. Pinto, M. D. Prokić, G. Arsa, and A. Vasconsuelo, "Oxidative stress in muscle diseases: current and future therapy 2019," Oxidative Medicine and Cellular Longevity, vol. 2020, Article ID 6030417, 4 pages, 2020.

[2] S. F. Vatner, J. Zhang, M. Oydanich, T. Berkman, R. Naftalovich, and D. E. Vatner, "Healthful aging mediated by inhibition of oxidative stress," Ageing Research Reviews, vol. 64, p. 101194, 2020.

[3] C. Wilson, "Oxidative stress and osteoporosis," Nature Reviews Endocrinology, vol. 10, no. 1, p. 3, 2014.

[4] J. Lean, B. Kirstein, Z. Urry, T. Chambers, and K. Fuller, "Thioredoxin-1 mediates osteoclast stimulation by reactive oxygen species," Biochemical and Biophysical Research Communications, vol. 321, no. 4, pp. 845-850, 2004.

[5] A. M. Craft, J. S. Rockel, Y. Nartiss, R. A. Kandel, B. A. Alman, and G. M. Keller, "Generation of articular chondrocytes from human pluripotent stem cells," Nature Biotechnology, vol. 33, no. 6, pp. 638-645, 2015.

[6] W. K. Ong and S. Sugii, "Adipose-derived stem cells: fatty potentials for therapy," The International Journal of Biochemistry \& Cell Biology, vol. 45, no. 6, pp. 1083-1086, 2013.

[7] D. Rana, S. Kumar, T. J. Webster, and M. Ramalingam, "Impact of induced pluripotent stem cells in bone repair and regeneration," Current Osteoporosis Reports, vol. 17, no. 4, pp. 226-234, 2019.

[8] X. Fu, G. Liu, A. Halim, Y. Ju, Q. Luo, and A. G. Song, "Mesenchymal stem cell migration and tissue repair," Cell, vol. 8 , no. 8, p. 784, 2019.

[9] Y. Liu, A. D. Berendsen, S. Jia et al., "Intracellular VEGF regulates the balance between osteoblast and adipocyte differentiation," The Journal of Clinical Investigation, vol. 122, no. 9, pp. 3101-3113, 2012.

[10] D. Zhang, N. Ni, Y. Wang et al., "CircRNA-vgll3 promotes osteogenic differentiation of adipose-derived mesenchymal stem cells via modulating miRNA-dependent integrin $\alpha 5$ 
expression," Cell Death and Differentiation, vol. 28, no. 1, pp. 283-302, 2021.

[11] S. Neri and R. Borzì, "Molecular mechanisms contributing to mesenchymal stromal cell aging," Biomolecules, vol. 10, no. 2, p. 340, 2020.

[12] M. C. Gómez-Puerto, L. P. Verhagen, A. K. Braat, E. W. F. Lam, P. J. Coffer, and M. J. Lorenowicz, "Activation of autophagy by FOXO3 regulates redox homeostasis during osteogenic differentiation," Autophagy, vol. 12, no. 10, pp. 1804-1816, 2016.

[13] R. Yang, J. Chen, J. Zhang et al., "1,25-Dihydroxyvitamin D protects against age-related osteoporosis by a novel VDREzh2-p16 signal axis," Aging Cell, vol. 19, no. 2, article e13095, 2020.

[14] W. Sun, W. Qiao, B. Zhou et al., "Overexpression of Sirt1 in mesenchymal stem cells protects against bone loss in mice by FOXO3a deacetylation and oxidative stress inhibition," Metabolism, vol. 88, pp. 61-71, 2018.

[15] F. Zhang, W. Peng, J. Zhang et al., "P53 and Parkin co-regulate mitophagy in bone marrow mesenchymal stem cells to promote the repair of early steroid-induced osteonecrosis of the femoral head," Cell Death \& Disease, vol. 11, no. 1, p. 42, 2020.

[16] T. X. Lu and M. E. Rothenberg, "MicroRNA," The Journal of Allergy and Clinical Immunology, vol. 141, no. 4, pp. 12021207, 2018.

[17] X. B. Qi, B. Jia, W. Wang et al., "Role of miR-199a-5p in osteoblast differentiation by targeting TET2," Gene, vol. 726, p. 144193, 2020.

[18] L. Fan, J. Fan, Y. Liu et al., "miR-450b promotes osteogenic differentiation in vitro and enhances bone formation in vivo by targetingBMP3," Stem Cells and Development, vol. 27, no. 9, pp. 600-611, 2018.

[19] Z. J. Zhang, H. Zhang, Y. Kang et al., "miRNA expression profile during osteogenic differentiation of human adiposederived stem cells," Journal of Cellular Biochemistry, vol. 113, no. 3, pp. 888-898, 2012.

[20] E. K. Moioli, L. Hong, and J. J. Mao, "Inhibition of osteogenic differentiation of human mesenchymal stem cells," Wound Repair and Regeneration, vol. 15, no. 3, pp. 413-421, 2007.

[21] R. T. Qomi and M. Sheykhhasan, "Adipose-derived stromal cell in regenerative medicine: a review," World Journal of Stem Cells, vol. 9, no. 8, pp. 107-117, 2017.

[22] S. Mollazadeh, B. S. Fazly Bazzaz, V. Neshati et al., "T-Box20 inhibits osteogenic differentiation in adipose-derived human mesenchymal stem cells: the role of T-Box20 on osteogenesis," Journal of Biological Research (Thessaloniki), vol. 26, no. 1, 2019.

[23] E. Chiarella, A. Aloisio, B. Codispoti et al., "ZNF521 has an inhibitory effect on the adipogenic differentiation of human adipose-derived mesenchymal stem cells," Stem Cell Reviews and Reports, vol. 14, no. 6, pp. 901-914, 2018.

[24] A. Narayanan, N. Srinaath, M. Rohini, and N. Selvamurugan, "Regulation of Runx2 by microRNAs in osteoblast differentiation," Life Sciences, vol. 232, p. 116676, 2019.

[25] Z. Yuan, Q. Li, S. Luo et al., "PPAR $\gamma$; and Wnt signaling in adipogenic and osteogenic differentiation of mesenchymal stem cells," Current Stem Cell Research \& Therapy, vol. 11, no. 3, pp. 216-225, 2016.

[26] Y. Zhang, D. Zhang, J. Lv, S. Wang, and Q. Zhang, "MiR-125a$5 \mathrm{p}$ suppresses bladder cancer progression through targeting FUT4," Biomedicine \& Pharmacotherapy, vol. 108, pp. 10391047, 2018.
[27] L. Sun, J. X. Lian, and S. Meng, "MiR-125a-5p promotes osteoclastogenesis by targeting TNFRSF1B," Cellular \& Molecular Biology Letters, vol. 24, no. 1, p. 23, 2019.

[28] S. Capasso, N. Alessio, T. Squillaro et al., "Changes in autophagy, proteasome activity and metabolism to determine a specific signature for acute and chronic senescent mesenchymal stromal cells," Oncotarget, vol. 6, no. 37, pp. 39457-39468, 2015.

[29] Y. B. Zhang, Z. M. Zhong, G. Hou, H. Jiang, and J. T. Chen, "Involvement of oxidative stress in age-related bone loss," The Journal of Surgical Research, vol. 169, no. 1, pp. e37-e42, 2011.

[30] L. Rochette, L. Mazini, G. Malka, M. Zeller, Y. Cottin, and C. Vergely, "The crosstalk of adipose-derived stem cells (ADSC), oxidative stress, and inflammation in protective and adaptive responses," International Journal of Molecular Sciences, vol. 21, no. 23, p. 9262, 2020.

[31] R. M. H. Rumney, S. A. Lanham, J. M. Kanczler et al., "In vivo delivery of VEGF RNA and protein to increase osteogenesis and intraosseous angiogenesis," Scientific Reports, vol. 9, no. 1, article 17745, 2019. 